$04,11,14$

\title{
Недебаевский диэлектрический отклик в монолитных слоях стеарата серебра
}

\author{
(C) Р.А. Кастро, М.А. Горяев, А.П. Смирнов \\ Российский государственный педагогический университет им. А.И. Герцена, \\ Санкт-Петербург, Россия \\ "E-mail: smirnov_alexander_hspu@mail.ru \\ (Поступила в Редакцию 4 июля 2016 г.)
}

Представлены результаты исследования электрофизических свойств стеарата серебра методами диэлектрической спектроскопии и дифференциальной сканирующей калориметрии (ДСК). Установлены механизмы переноса заряда и диэлектрической релаксации в стеарате серебра. Обнаружено существование критической температуры $T=338 \mathrm{~K}$, вероятно связанной с низкотемпературным фазовым переходом в исследуемой системе.

Работа выполнена при поддержке Минобрнауки РФ в рамках выполнения госзадания № 2014/376 (базовая часть).

DOI: $10.21883 /$ FTT.2017.02.44044.278

\section{1. Введение}

В настоящее время для оперативной регистрации оптических изображений широко применяются композиты на основе фототермографических материалов. Основными компонентами данных композитов являются галогениды серебра и соли жирных кислот, в частности стеарат серебра. При изготовлении светочувствительной композиции на поверхности частицы соли жирной кислоты (стеарата серебра) синтезируется галогенид серебра $[1,2]$. С помощью различных красителей-сенсибилизаторов, вводимых в подобную термопроявляемую композицию, можно провести спектральную сенсибилизацию фототермографических материалов, т.е. сделать чувствительными практически к любой области спектра от синей до ближней инфракрасной [3]. Стеарат серебра, являющийся одной из основных компонент фототермографических материалов, играет важную роль как в процессе сенсибилизации, так и в процессе проявления скрытого изображения $[4,5]$. Следовательно, изучение электрических и оптических свойств, а также выявление структурных особенностей данного материала, является актуальной задачей. Целью данной работы являлось выявление особенностей процессов диэлектрической релаксации и переноса заряда в слоях стеарата серебра, их связи со структурными особенностями материала. Известно, что структурные особенности неупорядоченных систем обусловлены существованием в них дефектных электронных или примесных центров [6,7]. Оценка влияния таких центров на электрофизические свойства материалов позволяет выявить природу процессов, протекающих в них, и расширить области их практического применения.

\section{2. Методика эксперимента}

Стеарат серебра получают в результате обменной реакции замещения натрия при избыточной концентрации нитрата серебра в виде частиц размером около микрона $[4,8]$, поэтому методом прессовки изготавливают монолитные образцы данного вещества в форме таблетки. Диаметр образцов был равен $10 \mathrm{~mm}$, толщина образцов - $0.5 \mathrm{~mm}$. После изготовления образцов было проведено исследование их элементного состава на сканирующем электронном микроскопе (СЭM) Zeiss EVO 40. В табл. 1 указан элементный состав исследуемых образцов, полученный методом сканирующей электронной микроскопии (СЭМ).

Особенности процессов диэлектрической релаксации и переноса заряда стеарата серебра исследовались методом диэлектрической спектроскопии на установке фирмы Novocontrol Technologies „Concept 41“. Данный метод успешно применяется при изучении электронных процессов в системах с различной степенью структурной неупорядоченности [9-11]. Установка „Concept 41“ включает в себя плату сопряжения с персональным компьютером и программное обеспечение для сбора данных. В качестве экспериментальных данных собирались значения мнимой и действительной части импеданса ячейки с измеряемым образцом. Спектры комплексной диэлектрической проницаемости и комплексной проводимости рассчитывались из спектров импеданса по следующим формулам

$$
\varepsilon^{*}=\varepsilon^{\prime}-i \varepsilon^{\prime \prime}=-i /\left(\omega Z^{*}(\omega) C_{0}\right)
$$

где $C_{0}=\varepsilon_{0} S / d-$ емкость пустой ячейки. Комплексная проводимость выражается через импеданс

$$
\sigma^{*}=\sigma^{\prime}-i \sigma^{\prime \prime}=-i S /\left(\omega Z^{*}(\omega) d\right)
$$


Таблица 1. Элементный состав исследуемых образцов, полученный на СЭМ

\begin{tabular}{c|c|c|c|c|c}
\hline Элемент & $\begin{array}{c}\text { Условная } \\
\text { концентрации }\end{array}$ & $\begin{array}{c}\text { Массовая } \\
\text { доля }\end{array}$ & Весовой, \% & $\begin{array}{c}\text { Весовой, \% } \\
\text { Сигма }\end{array}$ & $\begin{array}{c}\text { Атомная } \\
\text { доля, \% }\end{array}$ \\
\hline $\mathrm{C}$ & 71.66 & 1.9577 & 70.52 & 0.19 & 85.41 \\
$\mathrm{O}$ & 4.67 & 0.6562 & 13.70 & 0.18 & 12.46 \\
$\mathrm{Ag}$ & 5.97 & 0.7294 & 15.78 & 0.14 & 2.13 \\
Итоги & & & 100 & &
\end{tabular}

Измерение температурно-частотной зависимости диэлектрических параметров образцов стеарата серебра производилось в широком интервале частот $\left(10^{-2}-10^{2} \mathrm{~Hz}\right)$ и температурном интервале от 273 до $353 \mathrm{~K}$. Рабочее напряжение составляло $1.0 \mathrm{~V}$. Погрешность измерения физических величин и расчета физических параметров не превышала $3 \%$.

\section{3. Результаты и их обсуждение}

На рис. 1 изображена частотная зависимость действительной части комплексной проводимости при различных температурах. По характеру поведения зависимости видно, что удельная проводимость слоев стеарата серебра увеличивается с ростом частоты по закону $\sigma=A \omega^{s}$, где $A-$ постоянная, $\omega-$ циклическая частота, $s-$ показатель степени.

По поведению данной зависимости можно сделать вывод о том, что с ростом температуры образец стеарата серебра проявляет себя как полимеры, в которых преобладает ионный тип проводимости [12]. На рис. 2 изображена температурная зависимость показателя степени $s$, который принимает значения $s=0.30-0.90$. При этом можно выделить два участка - слабой зависимости $(T=273-303 \mathrm{~K})$ и сильной зависимости $(T=313-353 \mathrm{~K})$. Обнаруженные закономерности являются признаком возможного существовании в стеарате серебра прыжкового механизма проводимости [13].

Согласно представлению Остина-Мотта [14], проводимость на переменном токе в неупорядоченных системах обусловлена прыжками электронов между парами локализованных состояний на уровне Ферми. В модели прыжка со случайной энергией [15] предполагается, что элементарным актом поляризации является надбарьерный переход одного электрона с одного состояния на другое, а энергия активации центра определена кулоновским взаимодействием. При этом значение показателя степени $s$ находится в интервале от 0.70 до 1.00 при комнатной температуре и падает с ростом температуры. Как известно, проводимость связана с плотностью локализованных состояний $N\left(E_{F}\right)$ на уровне Ферми через соотношение

$$
\sigma(\omega)=(\pi / 3)\left[N\left(E_{F}\right)\right]^{2} k_{B} T e^{2} \alpha^{-5} \omega\left[\ln \left(v_{p h} / \omega\right)\right]^{4},
$$

где $\alpha-$ параметр затухания волновых функций локализованных состояний, $v_{p h}-$ фононная частота. При- нимая значения $v_{p h}=10^{12} \mathrm{~Hz}, \alpha^{-1}=10 \mathrm{~A}^{0}$, на частоте $\omega=10^{-2} \mathrm{~Hz}$ при температуре $T=293 \mathrm{~K}$, получаем значение плотности локализованных состояний на уровне Ферми равной $N\left(E_{F}\right)=3.38 \cdot 10^{16} \mathrm{eV}^{-1} \cdot \mathrm{cm}^{-1}$.

По виду температурной зависимости удельной проводимости $\sigma^{\prime}$ (рис. 3) можно заключить, что перенос заряда в исследуемых структурах является термически активированным процессом. Обнаружена экспоненциальная зависимость $\sigma^{\prime}$ от температуры с наличием двух участков с энергиями активации $E_{a 1}=0.10 \mathrm{eV}$ и $E_{a 2}=0.48 \mathrm{eV}$ соответственно.

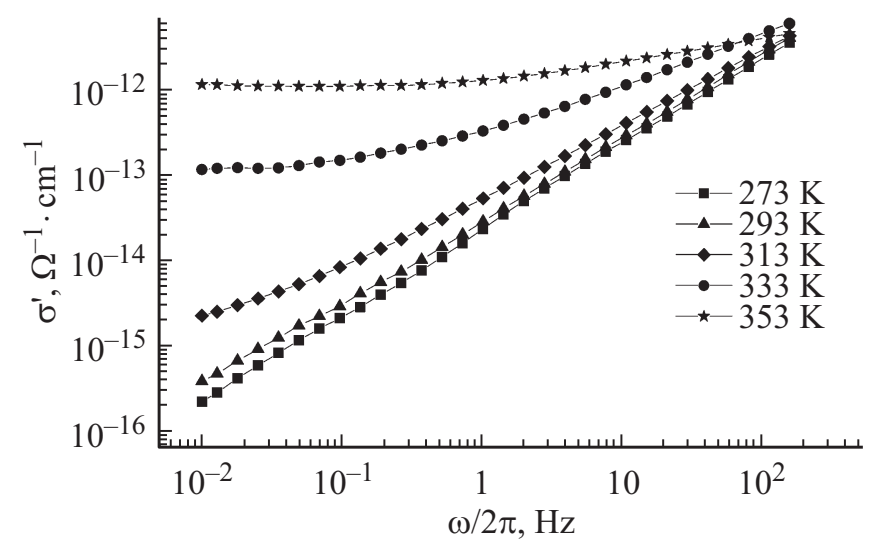

Рис. 1. Частотная зависимость удельной проводимости стеарата серебра при различных температурах.

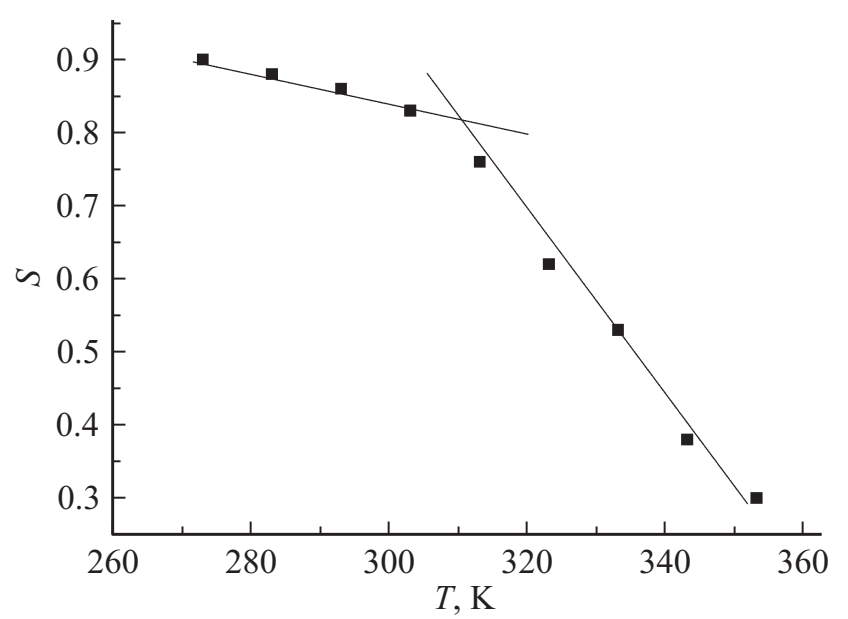

Рис. 2. Температурная зависимость показателя степени $s$. 


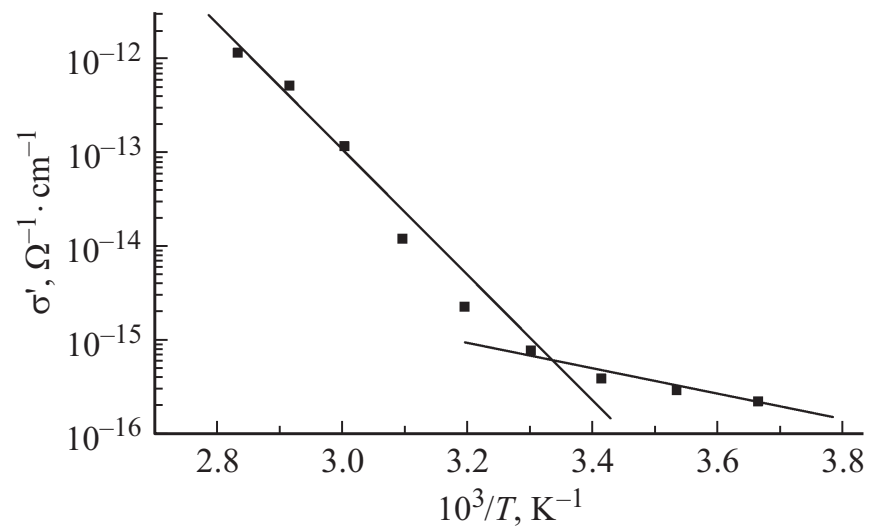

Рис. 3. Температурная зависимость натурального логарифма удельной проводимости при частоте $10^{-2} \mathrm{~Hz}$.

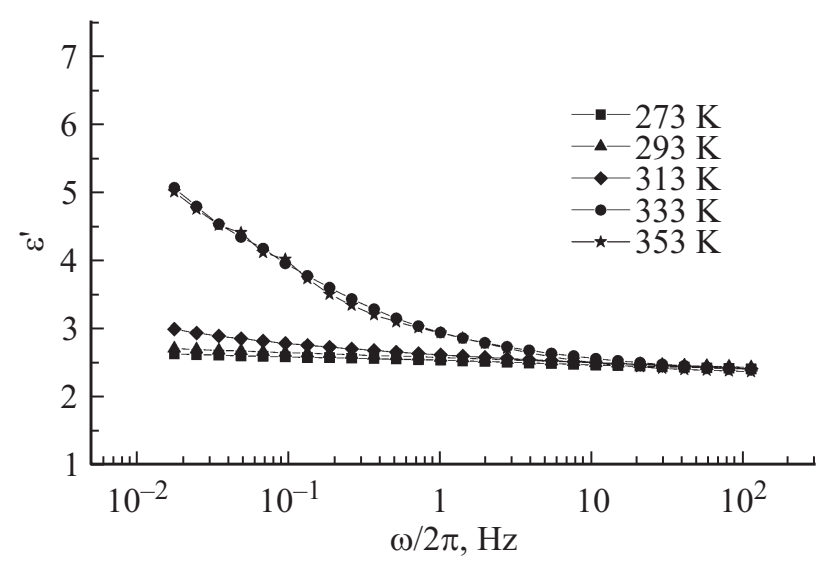

Рис. 4. Частотная зависимость диэлектрической проницаемости $\varepsilon^{\prime}$ слоев стеарата серебра при различных температурах.

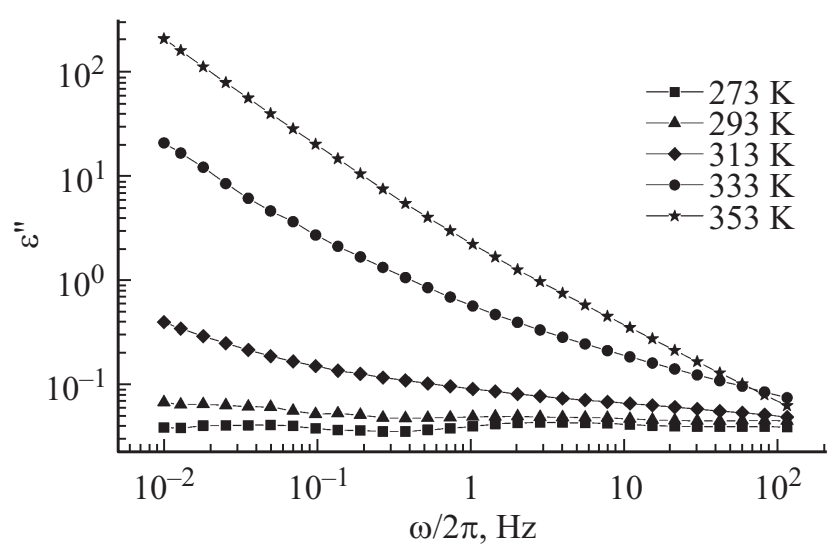

Рис. 5. Частотная зависимость фактора диэлектрических потерь $\varepsilon^{\prime \prime}$ в слоях стеарата серебра при различных температурах.

На рис. 4 и 5 представлены частотные зависимости составляющих комплексной диэлектрической проницаемости $\varepsilon^{\prime}$ и $\varepsilon^{\prime \prime}$ соответственно при различных температурах.

Частотная зависимость диэлектрической проницаемости $\varepsilon^{\prime}$ характеризуется наличием слабой дисперсии, которая становится заметнее в области более низких частот. С учетом структурных особенностей стеарата серебра, наличия в нем квазидипольных образований в виде карбоксильных хвостов и углеводородных цепей, можно заключить, что определяющим механизмом поляризации в исследуемом интервале частот является дипольно-релаксационная поляризация [12]. Рост диэлектрической проницаемости с температурой может быть объяснен связью механизма дипольной поляризации с тепловым движением молекул, вследствие чего диполи слабо ориентируются при низких температурах. В нашем случае наблюдается резкое увеличение значения $\varepsilon^{\prime}$ с ростом температуры начиная с $T=333 \mathrm{~K}$.

Существование температуры, при которой меняется характер температурной и частотной зависимостей удельной проводимости и диэлектрической проницаемости, указывает либо на возможное существование низкотемпературного фазового перехода, либо на начало температурной области, связанной с первым высокотемпературным переходом при $T=398 \mathrm{~K}$ (переход кристаллcurd) [13]. Для более определенного объяснения данного обстоятельства образец стеарата серебра исследовался методом ДСК. На рис. 6 изображена кривая ДСК стеарата серебра.

На данном рисунке в районе $T=338 \mathrm{~K}$ отчетливо виден эндотермический пик, который, вероятно, свидетельствует о наличии фазового перехода в стеарате серебра при данной температуре. Также на данной кривой отчетливо виден второй эндотермический пик, соответствующий уже известному фазовому переходу кристаллcurd $[11,16]$, который происходит при $T=397 \mathrm{~K}$.

Обнаруженная дисперсия фактора потерь $\varepsilon^{\prime \prime}$ (рис. 7), скорее всего, связана с проявлением механизма релаксационных потерь. Рост диэлектрических потерь с температурой может быть объяснен ростом вклада потерь проводимости при увеличении температуры образцов.

Во многих диэлектриках релаксационные процессы связаны с существованием не одного времени релаксации, а с набором времен релаксации. В таком случае

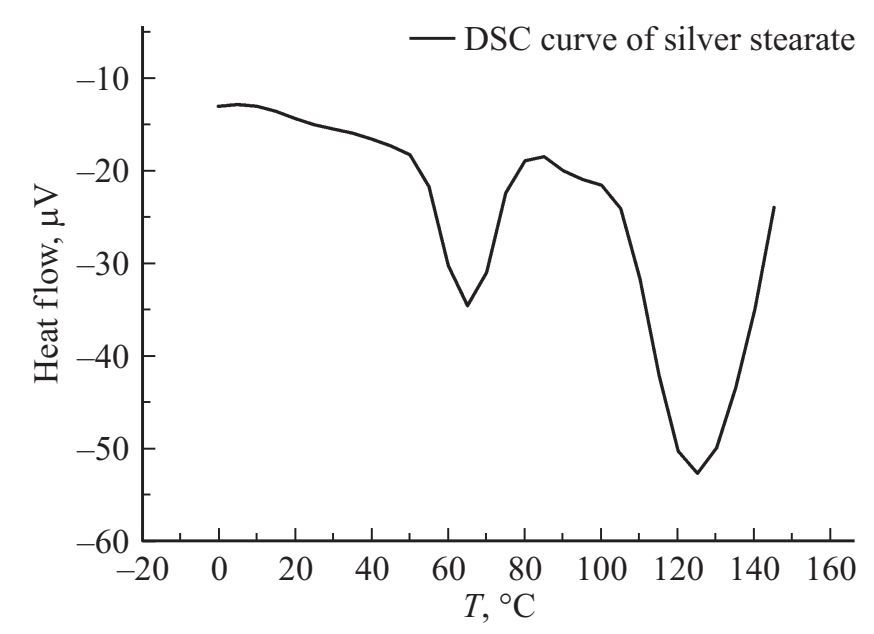

Рис. 6. Кривая ДСК исследуемого образца стеарата серебра. 
Таблица 2. Значение релаксационных параметров образцов стеарата серебра в области низких частот

\begin{tabular}{c|c|c|c|c|c}
\hline$T(\mathrm{~K})$ & $\tau_{\max }(\mathrm{s})$ & $\tau_{H N}(\mathrm{~s})$ & $\Delta \varepsilon$ & $\alpha_{H N}$ & $\beta_{H N}$ \\
\hline 273 & $7.20 \cdot 10^{-3}$ & $7.20 \cdot 10^{-3}$ & $3.74 \cdot 10^{-1}$ & 0.27 & 1.00 \\
283 & $3.93 \cdot 10^{-3}$ & $3.93 \cdot 10^{-3}$ & $3.32 \cdot 10^{-1}$ & 0.29 & 1.00 \\
293 & $2.91 \cdot 10^{-3}$ & $2.91 \cdot 10^{-3}$ & $2.97 \cdot 10^{-1}$ & 0.32 & 1.00 \\
303 & $1.06 \cdot 10^{-2}$ & $1.06 \cdot 10^{-2}$ & $4.21 \cdot 10^{-1}$ & 0.30 & 1.00 \\
313 & $1.45 \cdot 10^{-1}$ & $1.45 \cdot 10^{-1}$ & $6.42 \cdot 10^{-1}$ & 0.28 & 1.00
\end{tabular}

можно говорить о наличии распределения времени релаксации и, следовательно, энергий активации. Данное распределение может быть связано с проявлением различных по природе релаксационных процессов, или с распределением по концентрации диполей в структуре. В случае ионных прыжковых процессов предполагается, что после каждого прыжка меняется потенциальная энергия и необходимо некоторое время для возвращения к минимуму потенциальной энергии. Если учесть вклад большого количества подвижных дефектов, то получаем набор времен релаксации [12].

Отклонение от классической модели релаксации по Дебаю для случая одного времени релаксации можно исследовать, используя модельные представления Коула-Коула или Коула-Дэвидсона для случая симметричного и несимметричного распределения времен релаксации соответственно. Если построить диаграмму Коула-Коула (зависимость $\varepsilon^{\prime \prime}=f\left(\varepsilon^{\prime}\right)$ ), то существование распределения времен релаксации будет проявляться в отклонении от полусферической зависимости с радиусом полусферы $\left(\varepsilon_{s}-\varepsilon_{\infty}\right) / 2$ (рис. 7).

Существование недебаевского механизма релаксации подтверждается при оценке значений параметров в двухпараметрической эмпирической функции Гавриляк-Негами $(H N)$ [12]. Результаты измерения диэлектрических спектров аппроксимировались с использованием программного обеспечения WinFit 3.3 (Novocontrol Technologies $\mathrm{GmbH}$ ) (рис. 8). На основе этой аппроксимации выделялись положения максимумов диэлектрических потерь и вклада проводимости, определялись параметры $(H N)$ для изученных поляризационных процессов согласно выражению

$$
\varepsilon^{*}(\omega)=\varepsilon_{\infty}+\Delta \varepsilon /\left[1+(i \omega \tau)^{\alpha_{H N}}\right]^{\beta_{H N}},
$$

где $\varepsilon_{\infty}-$ высокочастотный предел действительной части диэлектрической проницаемости, $\Delta \varepsilon-$ диэлектрический инкремент (разность между низкочастотным и высокочастотным пределами), $\omega=2 \pi f, \alpha_{H N}$ и $\beta_{H N}$ - параметры формы, описывающие соответственно симметричное $(\beta=1.00$ - распределение Коула-Коула) и асимметричное $(\alpha=1.00$ - распределение Коула-Дэвидсона) расширение функции релаксации. Значения параметров, полученные при аппроксимации релаксационных кривых, представлены в табл. 2.
C учетом погрешности аппроксимации можно заключить, что в исследуемом интервале частот и температур в слоях стеарата серебра наблюдается дебаевский коле-

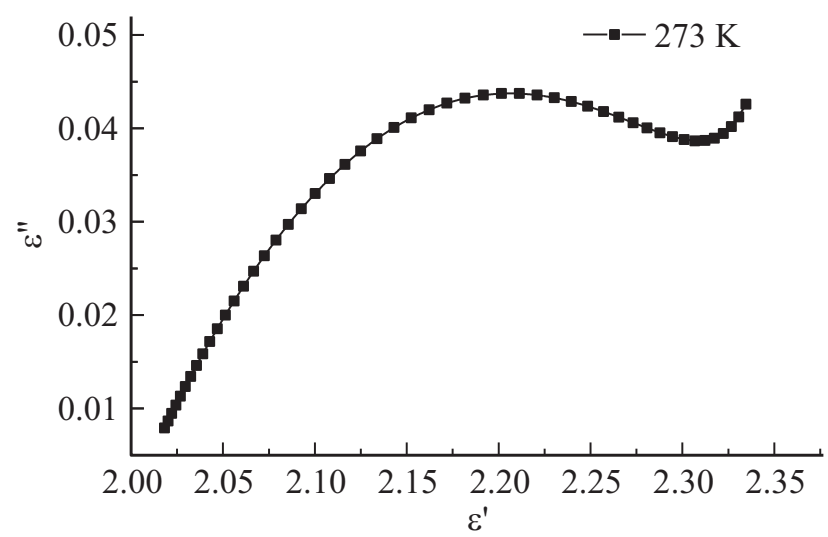

Рис. 7. Диаграмма Коула-Коула для образцов стеарата серебра при температуре $T=273 \mathrm{~K}$.

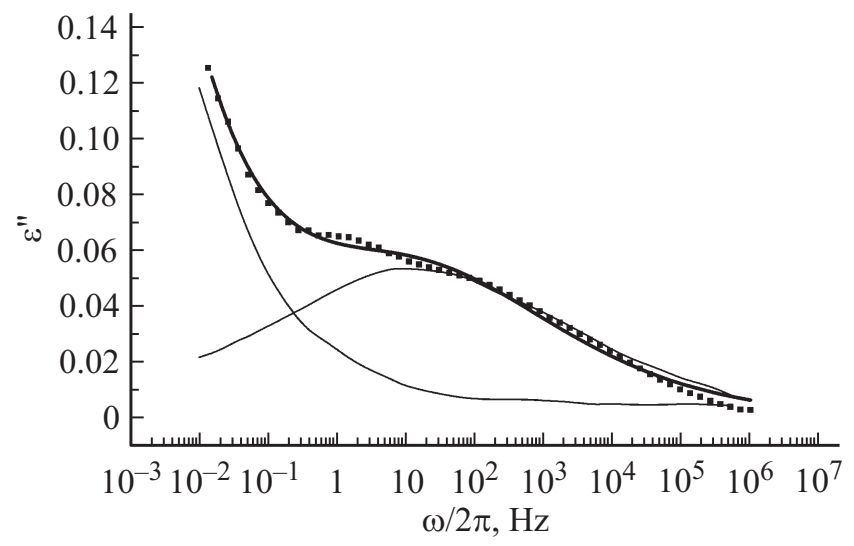

Рис. 8. Аппроксимация экспериментальной зависимости фактора потерь $\varepsilon^{\prime \prime}$ от частоты функцией $(H N)$ для температуры $T=303 \mathrm{~K}$.

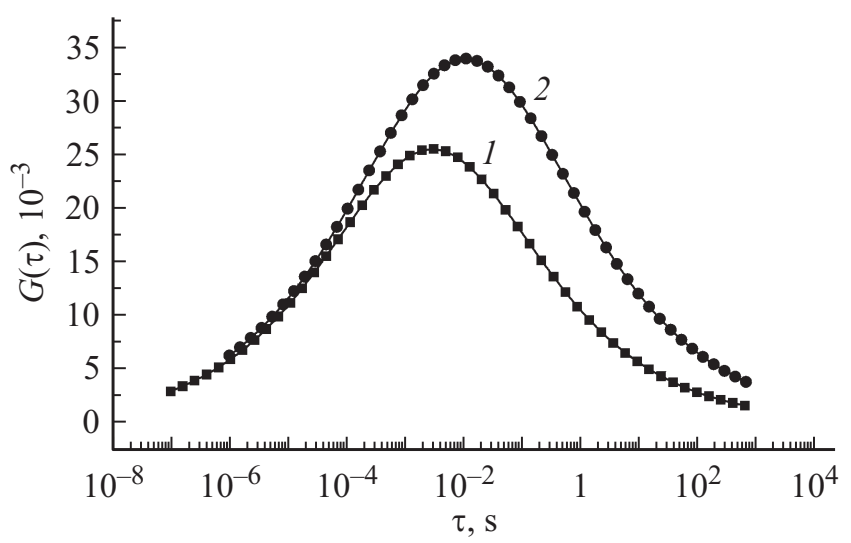

Рис. 9. Функция распределения релаксаторов по временам релаксации $G(\tau)$ в образцах стеарата серебра, восстановленная по значениям релаксационных параметров приближения $H N$ $\left(\alpha_{H N}, \beta_{H N}\right)$ при двух температурах T. $1-283,2-303 \mathrm{~K}$. 
бательный процесс с распределением времен релаксации согласно модели Коула-Коула. Увеличение значения наиболее вероятного времени релаксации $\tau_{\max }$ после температуры $T=303 \mathrm{~K}$ (рис. 9) подтверждает сделанное ранее предположение о существовании точки перехода (фазового перехода или начала этого процесса).

\section{4. Заключение}

В рамках проведенного исследования процессов диэлектрической релаксации и переноса заряда в монолитных слоях стеарата серебра обнаружены следующие закономерности.

1. Частотная зависимость действительной части удельной проводимости имеет вид $\sigma=A \omega^{s}$, что свидетельствует о существовании прыжкового механизма проводимости по локализованным состояниям. Температурная зависимость $\sigma$ характеризуется наличием двух участков с различными энергиями активации.

2. Увеличение диэлектрической проницаемости с уменьшением частоты и ростом температуры, а также увеличение величины диэлектрических потерь с температурой, скорее всего, связаны с проявлением механизмов дипольно-релаксационной поляризации.

3. Вид диграммы Коула-Коула и анализ релаксационных кривых в рамках приближения Гавриля-Негами позволяют сделать вывод о том, что стеарат серебра ведет себя как недебаевский диэлектрик, для которого характерно существование широкого распределения релаксаторов по временам релаксации.

4. Обнаружено существование температуры, при которой меняется характер дисперсии диэлектрических параметров. Кроме того, при этой же температуре обнаружен эндотермический пик на кривой ДСК стеарата серебра. Данные факты указывают на возможное существование низкотемпературного фазового перехода.

\section{Список литературы}

[1] М.А. Горяев. Журн. научн. и прикл. фотогр. и кинематогр. 36, 5, 421 (1991).

[2] D.A. Morgan. Phot. Sci. 41, 1, 108 (1993).

[3] М.А. Горяев, Б.И. Шапиро. Журн. научн. и прикл. фотогр. 42, 2, 65 (1997).

[4] М.А. Горяев. Изд-во РГПУ 306 (2011).

[5] М.А. Горяев, А.П. Смирнов. Изд-во РГПУ 144. 29 (2012).

[6] Р.А. Кастро, С.А. Немов, П.П. Серегин. ФТП 40, 927 (2006); [Semiconductors 40, 8, 898 (2006)].

[7] N.I. Anisimova, G.A. Bordovsky, V.A. Bordovsky, R.A. Castro. Radiation Effects Defects Solids 156, 359 (2001).

[8] М.А. Горяев. Письма в ЖТФ 20, 21, 40 (1994).

[9] R.A. Castro, G.A. Bordovsky, V.A. Bordovsky, N.I. Anisimova. J. Non-Crystal. Solids 352, 9-20, 1560 (2006).

[10] R.A. Castro, V.A. Bordovsky, G.I. Grabko. Glass Phys. Chem. 35, 1, 43 (2009).
[11] V.P. Afanasjev, I.B. Vendik, O.G. Vendig, N.Yu. Medevedeva, M.A. Odit, M.F. Sitnikova, A.A. Petrov, I.M. Sokolova, D.A. Chigirev, R.A. Castro. Glass Phys. Chem. 38, 1. 63 (2012).

[12] Broadband dielectric spectroscopy / Eds K. Kremer, A. Schonhals. Springer, Berlin Heidelberg (2003). 729 p.

[13] M.A. Goryaev, R.A. Castro, A.P. Smirnov. J. Phys. Conf. ser. 572, 012041. DOI:10.1088/1742-6596/572/1/012041. (2014).

[14] J.G. Austin, N.F. Mott. Adv. Phys. 18, 71, 41 (1969).

[15] М.С. Гутенев. Физ. и хим. стекла 9, 3, 291 (1983).

[16] S.J. Lee, S.W. Han, H.J. Choi, K. Kim. J. Phys. Chem. 106, 2892 (2002). 